\title{
IMMUNONUTRITION IN CERVICAL CANCER: IMMUNE RESPONSE MODULATION BY DIET
}

\author{
Oscar Medina-Contreras ${ }^{1}$, Julissa luvián-Morales ${ }^{2,3}$, Fernanda Valdez-Palomares $^{4,5}$, \\ Laura Flores-Cisneros ${ }^{2,3}$, Miriam S. SánChez-López ${ }^{2}$, Jaime H. SOto-Lugo ${ }^{6}$, AND Denisse \\ CASTRO-EGUILUZ ${ }^{7 *}$
}

\begin{abstract}
${ }^{1}$ Epidemiology, Endocrinology \& Nutrition Research Unit, Hospital Infantil de México Federico Gómez, Mexico City; 2Department of Clinical Research, Instituto Nacional de Cancerología (INCan), Mexico City; ${ }^{3}$ Medical Sciences Postgraduate Program, Universidad Nacional Autónoma de México (UNAM), Mexico City; ${ }^{4}$ Scientific Liaison Unit, Instituto Nacional de Medicina Genómica, Mexico City; ${ }^{5}$ Experimental Clinical Research Postgraduate Program, UNAM, Mexico City; ${ }^{6}$ Department of Radiation Oncology, Christus Muguerza, Hospital del Parque, Chihuahua, Chih.; ${ }^{7}$ Consejo Nacional de Ciencia y Tecnología (CONACyT)- Department of Clinical Research, INCan, Mexico City, Mexico
\end{abstract}

\begin{abstract}
In the development of cervical cancer (CC), the immune response plays an essential role, from the elimination of human papillomavirus (HPV) infection to the response against the tumor. For optimal function of the immune response, various factors are required, one of the most important being an adequate nutrition. The complex interaction between nutrients and microbiota maintains the immune system in homeostasis and in case of infection, it provides the ability to fight against pathogen invasion, as occurs in HPV infection. The purpose of this article is to describe the role of diet, food, and specific nutrients in the immune response from the onset of infection to progression to precancerous lesions and CC, as well as the role of diet and nutrition during oncological treatment. The immunomodulatory role of microbiota is also discussed. A detailed analysis of the evidence leads us to recommend a nutritional pattern very similar to the Mediterranean diet or the prudent diet for an optimal immune response. Moreover, pre- and probiotics favorably modulate the microbiota and induce preventive and therapeutic effects against cancer. (REV INVEST CLIN. 2020;72(4):219-30)
\end{abstract}

Key words: Immunonutrition. Diet. Human papillomavirus. Cervical cancer. Microbiota.

\section{INTRODUCTION}

The optimal function of the immune system is crucial for health in general, for the prevention and elimination of infections, and in immune surveillance against tumor cells. Nutrition is one of the most important factors that modulate different aspects of immune function ${ }^{1}$. The ability of the immune system to identify and respond against cancer is determined by several factors, including the host's genetic makeup, the somatic profile of cancer cells and the environment. Nutrition is a fundamental environmental factor that
*Corresponding author:

Denisse Castro-Eguiluz

E-mail: angeldenisse@gmail.com
Received for publication: 20-02-2020

Approved for publication: 19-04-2020

DOI: $10.24875 / R I C .20000062$

0034-8376 / (C) 2020 Revista de Investigación Clínica. Published by Permanyer. This is an open access article under the CC BY-NC-ND license (http://creativecommons.org/licenses/by-nc-nd/4.0/). 
acts through systemic or local effects within the tumor microenvironment, by modulating cell metabolism pathways through specific nutrients, such as antioxidants, by immune system modulation, and intestinal microbiota regulation ${ }^{2}$.

The impact of nutrition on the immune system is an area that remains under investigation. However, studies conducted to date, provide compelling evidence that nutritional status and the immune system are strongly linked, and that integrity of the immune system can be rapidly altered by changes in the nutritional status ${ }^{3}$.

Immunonutrition is a discipline that studies the relationships between nutrition, immunity, infection, inflammation, injury, and healing. Its development has underscored the fact that with nutrition, it is possible to prevent disease in healthy individuals and to treat disease in compromised individuals; it has also allowed to analyze the effects of nutrition on the modulation of the innate and adaptive immune responses.

Nutrition is a determining factor in immune system responses, with malnutrition being the most common cause of immunodeficiency worldwide. Protein deficiency malnutrition has been associated with a significant decrease in cell-mediated immunity, phagocytic function, the complement system, secretion of immunoglobulin A antibodies, and cytokine production. In addition, the lack of some specific nutrients also results in disruption of the immune response, including micronutrients such as zinc, selenium, iron, copper, Vitamins A, C, E, and B-6, and folic acid ${ }^{4}$.

The diet can affect the microbial community of the gut, a complex and dynamic system, crucial for the development and maturation of both systemic and gut mucosal immune responses, and also plays an important role in metabolism, nutrition, and physiological characteristics. Therefore, the complex interaction between available nutrients, the bacterial community, and the immune system is the main regulator maintaining homeostasis; it establishes an effector response against pathogen invaders 5 and maintains immune surveillance for the elimination of malignant cells.

In this review the interaction of nutrition and immune response against human papillomavirus (HPV)infected and cervical cancer (CC) cells will be explained, along with the role that the microbiota have been thus far identified to play in this interaction. We will also discuss how therapeutic approaches have been directed against the tumor, but with adverse consequences on the nutritional status of individuals suffering from this disease. At present, novel targeted treatments, in particular, immunotherapies, are also being rapidly developed, although their effect on nutrition is yet to be assessed. For this review, the NCBI-PubMed database was used to search for original articles that investigated the effects and associations between specific nutrients, foods, and dietary patterns on infection with HPV, development of cervical intraepithelial neoplasia (CIN), CC, and cancer treatment. We also searched for articles that investigated the interaction of nutrition with the microbiota and its association with chronic HPV infection, the development of the disease and treatment-related toxicities. All authors participated in the search, reviewing (according to the GRADE system), and discussion of the articles included in this review, and elaborated recommendations accordingly.

\section{DIETARY PATTERNS, FOODS, AND NUTRIENTS ASSOCIATED WITH AN EFFICIENT IMMUNE RESPONSE AGAINST HPV}

Although HPV infection is a necessary condition for the development of CC, the presence of other factors is required for the infection to progress to cervical lesion and subsequently to cancer. Nutritional status can be an important factor, since a poor nutritional status can lead to failure of the immune response to eliminate the infection, thus favoring the persistence of infection, and the progression to CIN.

Other factors, such as malnutrition, obesity, visceral adiposity, and their metabolic and pro-inflammatory effects, have also been linked to the development of tumors ${ }^{6}$. In the case of CC, obesity (Body mass index $>30 \mathrm{~kg} / \mathrm{m}^{2}$ ) is a factor that increases the risk of developing this neoplasm by $10 \%$ (hazard ratio $[\mathrm{HR}]=1.10 ; 95 \%$ confidence interval $[\mathrm{Cl}]: 1.03$, $1.17)^{7}$. Excessive visceral adipose tissue induces a low-grade chronic inflammatory response, since it increases the secretion of inflammatory molecules, 
such as tumor necrosis factor-alpha, interferon-gamma, interleukin IL-6, and IL-1beta, in addition to modifying endogenous hormone metabolism ${ }^{8}$. This chronic inflammation has been associated with $25 \%$ of all tumors and implicated in the oncogenic process 9 .

An adequate diet that covers energy-protein and micronutrient requirements is essential to maintain the immune system in optimal function ${ }^{10}$. Therefore, including in the diet foods that are rich in some nutrients has led to a decrease in the risk of persistent HPV infection ${ }^{11}$. Further, the intake of Vitamins $A, C$, $E$, and $D$, and folate has been reported to help inhibit cell proliferation, prevent DNA damage, and enhance immune functions ${ }^{12}$.

The European Prospective Investigation into Cancer (EPIC) study analyzed the relationship between nutrition and the incidence of cancer. The study followed 142,605 men and 335,875 women from ten different countries over a period of 8.7 years. Overall, 9669 cases of cancer were identified in men and 21,062 cases in women. Adherence to the Mediterranean diet was associated with a decreased cancer risk, with a HR of $0.96(95 \% \mathrm{Cl}: 0.95-0.98)$ for all types of cancer $(p<0.0001)$. When considering the risk by food groups, an apparent protective effect of fruits and nuts, vegetables, grains, and a high unsaturated/saturated fatty acid ratio was observed. High meat consumption was associated with an increased risk of cancer, as well as moderate alcohol intake. Analyses of the different components of the diet on all types of cancer seem to indicate that the beneficial effect of the Mediterranean diet is not due to any specific component, but to the combined effect of a range of nutrients and other components offered by a diet rich in antioxidants, fiber and polyphenols, with a fatty acid profile that favors the consumption of omega- 3 fatty acids ${ }^{13}$.

Barchitta et al. ${ }^{14}$ conducted a cross-sectional study in which three dietary patterns and their possible association with the development of high-risk HPV infection were analyzed. The patterns compared were the western diet, the Mediterranean diet, and the prudent diet. The Mediterranean diet is characterized by the consumption of vegetables, fruits, olive oil, fish, legumes, and whole grains. The western diet pattern is characterized by a higher intake of sugars, refined flours, dressings, vegetable oil, snacks, and chips, among others. The prudent diet includes raw and cooked vegetables, fruits, olive oil, potatoes, and legumes, among others. The regression model performed to establish associations between dietary patterns - derived by principal component analysis of the dietary pattern - and the risk of high-risk HPV infection, showed that the Mediterranean diet was associated with a lower risk of high-risk HPV infection (odds ratio $[\mathrm{OR}]=0.79 ; 95 \% \mathrm{Cl}=0.66-0.96 ; \mathrm{p}=0.018$ ), whereas the western diet was associated with an increased risk of HPV infection (OR $=1.44 ; 95 \%$ $\mathrm{Cl}=1.03-2.03, \mathrm{p}=0.036$ ). No significant differences were found with the prudent diet.

If we compare the western against the Mediterranean diet, we can see that the western diet lacks an adequate supply of fiber, polyunsaturated fatty acids, vitamins, minerals, antioxidants, and other nutrients; conversely, it provides more saturated fatty acids and empty calories that contribute to the development of metabolic disease. At the time of HPV infection, an increase in the production of reactive oxygen and nitrogen species by innate immune cells generates an increase in oxidative stress and if there are insufficient antioxidant compounds (as is the case when an individual follows a western dietary pattern), this oxidative stress will condition a pro-oncogenic environment, and the infection will progress more efficiently to neoplastic transformation. Although specific nutrients have beneficial effects on health, it is highly likely that a diet with a wide diversity of nutrients would have a greater impact as a protective factor against infection than one single nutrient. However, as described below, some authors found associations between specific nutrients and persistent HPV infection.

In the study by Lopes et al. ${ }^{12}$, several dietary components were analyzed as potential risk factors for the persistence of oncogenic, non-oncogenic, and unclassified HPV infection. Individuals with persistent oncogenic HPV infection were observed to have a higher energy consumption ( $p=0.0051$ ), lower vitamin $A$ $(p=0002)$, and lower folate intakes $(p=0.0001)$. Individuals with persistent non-oncogenic HPV infection had a lower intake of Vitamin B12 $(p=0.0436)$. Those with persistent unclassified HPV infection had a decreased intake of alpha-carotene $(p=0.0241)$, beta-carotene $(p=0.0241)$, and lutein + zeaxanthin $(p=0.0403)$. 
Giuliano et al. ${ }^{15}$ conducted a cohort study with a follow-up period of 1 year. During follow-up, nutrient consumption was assessed: beta-cryptoxanthin $(\mathrm{OR}=0.47 ; 95 \% \mathrm{Cl}=0.26-0.85)$, lutein + zeaxanthin $(\mathrm{OR}=0.49 ; 95 \% \mathrm{Cl}=0.27-0.87)$, and vitamin $\mathrm{C}(\mathrm{OR}=0.5 ; 95 \% \mathrm{Cl}=0.27-0.92)$ were found to be dietary components that could be acting as protective factors, contributing to the elimination of HPV infection. Other nutrients associated with a lower risk of HPV infection were folate, retinol, lycopene, and Vitamin $A^{16}$. In addition, carrots and papaya (> $200 \mathrm{~g} /$ day) were associated with a lower risk of HPV persistent infection ${ }^{17}$. Resveratrol, a compound that is naturally found in some fruits and seeds, has been observed to be able to stimulate the immune response with an increase in natural killer cell-mediated elimination of virus-infected and cancer cells. Grapes and red wine are foods with the highest amount of resveratrol, followed by blackberries, currants, cranberries, nuts, and cocoa. Incorporation of resveratrol into the diet through these foods can have immunomodulatory effects ${ }^{18}$.

According to the results obtained, we can conclude that the factors which could limit the immune response during HPV infection are low fruit and vegetable intake (mainly those that contribute with components such as Vitamins $A$ and $C$, carotenes, cryptoxanthin, folate, lutein, zeaxanthin, and resveratrol), and excessive energy consumption, with the latter probably being related to overweight and obesity. This leads us to recommend an eating pattern very similar to the Mediterranean diet or the prudent diet.

\section{ROLE OF FOODS AND NUTRIENTS IN THE CONTROL AND ELIMINATION OF CIN}

The study by Barchitta et al. ${ }^{14}$ also analyzed whether the prudent diet pattern was associated with the development of $\mathrm{CIN} 2+$. The results showed that the prudent diet might be a protective factor against the development of $\mathrm{CIN} 2+(\mathrm{OR}=0.50 ; 95 \% \mathrm{Cl}=0.26-0.98$; $\mathrm{p}=0.039$ ). No significant differences were found with the western diet or with the Mediterranean diet.

Tomita et al. ${ }^{19}$ conducted a case-control study to determine if there was an association between dietary intake and newly diagnosed CIN1, CIN2, and
CIN3. In this study, the consumption of carrots (between $203 \mathrm{~g}$ and $1321 \mathrm{~g} /$ day) was found to likely be a protective factor against the development of CIN3 $(\mathrm{OR}=0.50 ; 95 \% \mathrm{Cl}=0.27-0.95)$. In addition, a blood sample was obtained to measure serum tocopherols and carotenes and analyzed their possible association with the development of CIN or invasive CC. The results showed that low serum levels of alpha-tocopherol, gamma-tocopherol, total carotenes, and lycopene increase the risk for developing CIN Grades 2 and 3 , and invasive CC.

Tomita et al. ${ }^{20}$ conducted another study in which the relationship between the presence of CIN3 and a limited consumption of five different food groups was analyzed. When analyzing all individuals, an association was observed between CIN3 and the consumption of $\leq 39 \mathrm{~g} /$ day of dark green and deep yellow fruits and vegetables $(\mathrm{OR}=1.71 ; 95 \% \mathrm{Cl}=1.15-2.52)$, as well as with an intake of $\leq 79 \mathrm{~g} /$ day of fruit and fruit juice $(\mathrm{OR}=1.51 ; 95 \% \mathrm{Cl}=1.05-2.17)$, consumption of $\leq 79 \mathrm{~g} /$ day of citrus fruits and citrus juices (OR $=1.44 ; 95 \% \mathrm{Cl}=1.02-2.03)$, and a total consumption of $\leq 319 \mathrm{~g} /$ day of fruits and vegetables $(O R=1.52 ; 1.06-2.17)$. When individuals were classified as smokers and non-smokers, only the first group ( $\leq 39 \mathrm{~g} /$ day of dark green and intense yellow fruits and vegetables) was found to remain as a risk factor in smokers $(\mathrm{OR}=1.96 ; 95 \% \mathrm{Cl}=1.15-3.33)$.

Previously, low levels of serum folate were reported to increase the risk of CIN progression ${ }^{21}$. Two hundred and forty-seven cases of cervical low-grade squamous intraepithelial lesions (LSIL), 125 cases of cervical high-grade squamous intraepithelial lesions (SIL) and 877 controls were analyzed. The increase in CIN was associated with higher rates of hrHPV infection and lower levels of serum folate. On the other hand, a study ${ }^{22}$ was conducted to determine the effects of long-term folate supplementation on regression and metabolic status of patients with CIN1. A higher percentage of women in the folate group developed lesion regression compared to the placebo group ( 83.3 vs. $52 \%, p=0.019$ ).

Although the evidence might seem to be inconsistent, a cohort study investigated the association between the consumption of fruits and vegetables within the EPIC study ${ }^{23}$. A total of 343,518 women from 23 different centers in Europe with a follow-up 
period of 9 years were included in the study. A statistically significant inverse association was found when fruit consumption was increased to $100 \mathrm{~g} /$ day (HR: $0.83 ; 95 \% \mathrm{Cl}: 0.72-0.98$ ) in women with invasive squamous cell CC; however, no significant association was found with the increase in vegetable consumption (100 g/day; HR: 0.85; 95\% Cl: 0.65-1.10).

In a study conducted in 390 women, the only dietary factors that were associated with the development of CIN Grades 2 and 3 were boiled coffee and dairy products ${ }^{24}$. Total fat, saturated, monounsaturated and polyunsaturated fatty acids, total fiber, fiber from grains, fiber from fruits and berries, fiber from vegetables, Vitamin C, and folate were also analyzed. Other foods associated with a lower risk of CIN progression were fruits in general, papaya, vegetables in general, onion, legumes, nuts, and a general intake of fruits and vegetables $>140 \mathrm{~g} /$ day. Consumption of $\leq 70 \mathrm{~g} /$ day of fruit and $\leq 39 \mathrm{~g} /$ day of dark-green fruits and vegetables was associated with a higher risk of CIN progression. Among the antioxidant compounds, polyphenols such as turmeric ${ }^{25-28}$, ferulic acid $^{29}$, epigallocatechin gallate ${ }^{29,30}$, and resveratrol ${ }^{30}$ have been found to possess antiviral, cytotoxic, antiinflammatory, and chemopreventive effects.

Other studies have also found an inverse relationship between blood antioxidant levels and the grade of cervical dysplasia. One study reported blood alpha-tocopherol levels of $(21.57 \mu \mathrm{g})$, compared to women with CIN1 $(21.18 \mu \mathrm{g}), \mathrm{CIN} 2(18.10 \mu \mathrm{g})$, and CIN3 $(17.27 \mu \mathrm{g})(p=0.012)^{15}$. In addition, higher consumption of papaya or orange is inversely related to the risk of developing cervical SIL ( $p=0.01$ and $p=0.02$, respectively), with the strongest association being with papaya consumption $>1$ times/ week $(\mathrm{OR}=0.19 ; 95 \% \mathrm{Cl}: 0.08-0.49)$ or orange consumption $>1$ times/week $(\mathrm{OR}=0.32 ; 95 \% \mathrm{Cl}$ : $0.12-0.87)^{31}$.

A study by Feng et al. ${ }^{32}$ assessed the consumption of certain food groups and their protective role in the development of $\mathrm{CIN}+$. Consumption of $\geq 15.95$ servings of onion per week was associated with a lower risk $(\mathrm{OR}=0.654 ; 95 \% \mathrm{Cl}=0.437-0.978 ; \mathrm{p}=0.036)$, as well as consumption of $\geq 2.69$ servings of legumes per week $(\mathrm{OR}=0.655 ; 95 \% \mathrm{Cl}=0.439-0.978 ; \mathrm{p}=0.038)$, consumption of $\geq 0.61$ servings of nuts per week (OR $=0.590 ; 95 \% \mathrm{Cl}=0.394-0.882 ; \mathrm{p}=0.008)$, and consumption of $\geq 0.94$ servings of meat per week $(\mathrm{OR}=0.651 ; 95 \% \mathrm{Cl}=0.429-0.987 ; \mathrm{p}=0.047)$.

Hwang et al. ${ }^{33}$ analyzed a cohort with a 3-year followup, to determine the relationship between the intake of fruits and vegetables and the development of cervical dysplasia (CIN1, CIN2, and CIN3) in HPV+ and HPV- subjects. Fruit consumption of $<109 \mathrm{~g} /$ day $(\mathrm{OR}=2.93 ; 95 \% \mathrm{Cl}=1.25-6.87 ; \mathrm{p}=0.01)$ and vegetable consumption of $<302 \mathrm{~g} /$ day $(\mathrm{OR}=2.84 ; 95 \%$ $\mathrm{Cl}=1.26-6.42 ; \mathrm{p}=0.06$ ) were found to be associated with CIN2 and CIN3 in HPV+ subjects.

We conclude that, for the control and elimination of $\mathrm{CIN}$, it is advisable to include in the diet a frequent intake of onions, legumes, nuts, and fruits and vegetables in general. Although meat would appear to be a protective factor (probably due to its Vitamin B12 content), increasing its consumption is not recommended due to its association with colon cancer. For the purposes of this review, a portion not exceeding $80 \mathrm{~g}$ of red meat per week is suggested.

\section{ROLE OF FOODS AND NUTRIENTS IN CERVICAL CANCER CARCINOGENESIS}

Inflammation is the central component of the innate immunity response. In general, inflammation is a local response to tissue injury characterized by an increase in blood flow, capillary dilation, leukocyte infiltration, and localized production of molecular mediators responsible for eliminating pathogens and repairing tissue injury. This inflammatory process is resolved with the effects of acute phase proteins, anti-inflammatory cytokines, and other anti-inflammatory components. However, a low-grade chronic inflammatory state is associated with a wide variety of chronic conditions, including the metabolic syndrome, steatohepatitis, type 2 diabetes mellitus, cardiovascular disease, and cancer ${ }^{34}$.

The correlation between chronic inflammation and cancer has been supported by epidemiological and experimental studies in humans and animals. Chronic inflammation plays a role in all cancer stages, increasing the rate of genetic mutations, and epigenetic mechanisms that lead to the onset of cancer, promoting tumor progression, and promoting a metastatic spread $^{35}$. 
Inflammation has been considered a predisposing factor to the development of tumors, with at least $20 \%$ of all types of cancer resulting from of a chronic inflammatory process ${ }^{36}$. Dietary intake plays a role in the physiological response to inflammation. Consequently, nutrition can influence both, the development and progression of inflammatory conditions and their prevention and treatment. Studies conducted to assess the relationship between dietary intake and low-grade inflammation have shown evidence that Mediterranean dietary patterns can be particularly beneficial in decreasing inflammation. In particular, the consumption of fruits, vegetables, and whole grains is associated with a decrease in the concentrations of C-reactive protein and fibrinogen, both inflammatory biomarkers.

Studies focusing on specific nutrients have shown that dietary antioxidants such as beta-carotene, zinc, selenium, Vitamin $\mathrm{C}$, and Vitamin $\mathrm{E}$ are associated with lower levels of inflammation markers. The effect of diet on HPV-16-induced carcinogenesis was tested in a study with nude mice fed a folaterich diet or a folate-deficient diet. The mice who consumed the folate-rich diet had a lower expression of oncogenic proteins E6 and E7 when compared to the mice that were fed a folate-deficient diet $^{21}$. Other non-nutritive components such as flavonoids, which can be found in fruits, vegetables, teas, coffee, red wine, and cocoa, also have high antioxidant power and anti-inflammatory activity ${ }^{2}$. Foods rich in omega- 3 fatty acids also help curb inflammation, since they are associated with a reduction in the production of pro-inflammatory cytokines and eicosanoids. In one study, after 2 weeks on a diet rich in omega- 3 fatty acids, study subjects showed a decrease in inflammatory mediators, which was maintained for 2 weeks after the dietary intervention ${ }^{37}$. In a population-based study, the consumption of foods rich in omega- 3 fatty acids has considerably decreased in the last 20 years ${ }^{38}$. Vitamin $E$ acts by reducing lipid peroxidation in cell membranes. If membrane lipid peroxidation is not controlled, it can generate free radicals that act as carcinogens. Besides exerting an antioxidant effect, Vitamin $E$ also decreases the neurotoxic effects of chemotherapy ${ }^{39}$.

In addition, studies in mice have shown that the use of prebiotics has anti-cancer and anti-inflammatory properties, since they modulate cyclooxygenase-2, nuclear transcription factor-kappa B (NF-kappa B), inducible nitric oxide synthase, and gastrointestinal glutathione peroxidase expression ${ }^{40,41}$.

Ghosh et al. ${ }^{42}$ conducted a case-control study, reporting that consumption of certain nutrients might be protective against the development of CC. These nutrients are: Polyunsaturated fatty acids $>12 \mathrm{~g} /$ day $(\mathrm{OR}=0.57 ; 95 \% \mathrm{Cl}=0.34-0.97 ; \mathrm{p}=0.04)$, fiber $>29 \mathrm{~g} /$ day $(\mathrm{OR}=0.59 ; 95 \% \mathrm{Cl}=0.37-0.94$; $\mathrm{p}=0.03)$, Vitamin $C>224 \mathrm{~g} /$ day $(O R=0.53 ; 95 \%$ $\mathrm{Cl}=0.33-0.8 ; \mathrm{p}<0.01)$, Vitamin $\mathrm{E}>8.9 \mathrm{mg} /$ day $(\mathrm{OR}=0.44 ; 95 \% \mathrm{Cl}=0.27-0.72 ; \mathrm{p}<0.01)$, Vitamin $A>12.7 \mathrm{IU} /$ day $(\mathrm{OR}=0.47 ; 95 \% \mathrm{Cl}=0.3-0.73$; $\mathrm{p}<0.01$ ), alpha-carotenes > $1.393 \mu \mathrm{g} /$ day $(\mathrm{OR}=0.41 ; 95 \% \mathrm{Cl}=0.27-0.63 ; \mathrm{p}<0.01)$, betacarotenes $>7.512 \mu \mathrm{g} /$ day $(\mathrm{OR}=0.44 ; 95 \%$ $\mathrm{Cl}=0.29-0.68 ; \mathrm{p}<0.01)$, lutein $(\mathrm{OR}=0.51 ; 95 \%$ $\mathrm{Cl}=0.33-0.79 ; \mathrm{p}<0.01$ ), lycopene $>5.837 \mu \mathrm{g} /$ day $(\mathrm{OR}=0.65 ; 95 \% \mathrm{Cl}=0.44-0.98 ; \mathrm{p}=0.04)$, and folate $>433.2 \mu \mathrm{g} /$ day $(\mathrm{OR}=0.55 ; 95 \% \mathrm{Cl}=0.34-0.88$; $\mathrm{p}=0.01$ ).

In a multicenter study by González et al. ${ }^{23}$, they searched for an association between the consumption of fruits and vegetables and the development of invasive squamous cell CC. Leafy vegetables $(\mathrm{HR}=0.52 ; 95 \% \mathrm{Cl}=0.29-0.95 ; \mathrm{p}=0.034)$, Vitamin $\mathrm{C}(\mathrm{HR}=0.59 ; 95 \% \mathrm{Cl}=0.39-0.89 ; \mathrm{p}=0.047)$, and Vitamin $D(H R=0.47 ; 95 \% \mathrm{Cl}=0.3-0.76 ; \mathrm{p}=0.004)$ were found to be protective factors. A case-control study by Hosono et al. ${ }^{43}$ was designed to determine if there is any association between calcium and $\mathrm{Vi}$ tamin $\mathrm{D}$ intake and the development of invasive cervical carcinoma. A calcium consumption of $\geq 502.6 \mathrm{mg} /$ day was observed to confer protection (OR $0.5 ; 95 \% \mathrm{Cl}=0.35-0.73 ; \mathrm{p}=0.004$ ). When the population was stratified by smoking status, the protective factor was observed to persist in nonsmokers $(p=0.006)$, as well as in patients with a squamous type histology ( $p=0.005)$; however, in the group of smokers $(p=0.796)$ and in patients with adenocarcinoma-type histology $(p=0.493)$, this protection is lost. Something very similar occurs with Vitamin $D$, where consumption of $\geq 162$ IU/day confers protection ( $p=0.014$ ), especially in non-smokers $(p=0.002)$ and in patients with squamous-type histology ( $p=0.017$ ); however, in smokers $(p=0.731)$ and in patients with 
adenocarcinoma-type histology $(p=0.423)$ this protection is lost.

The process of carcinogenesis takes years, which is why we insist in this review on the importance of nutritional education at all levels. The lack of compelling evidence generated in studies suggests that supplementation with a nutrient or multivitamins, or controlling dietary habits for a short period of time is not sufficient, since nutrient elements, including vitamins, minerals, antioxidants, and other components in food interact with each other, and this interaction might have an additive effect that could confer a state of protection. Studies that have investigated the Mediterranean diet agree that it decreases the risk of a variety of cancers by modulating multiple interconnected processes involved in carcinogenesis and in the inflammatory response, such as production of free radicals, NF-kappa B activation, and the expression of inflammatory mediators. In particular, the capability of this diet to induce an anti-inflammatory response has been described, as well as its usefulness in maintaining intestinal microbiota homeostasis and epigenetic oncogenesis modulation through specific microR$\mathrm{NAs}^{35}$. Finally, it is necessary to complement the diet with physical activity and to lead a healthy lifestyle. In addition, it should be noted that the protective effect provided by diet and lifestyle changes is blunted by smoking.

\section{ROLE OF NUTRITIONAL STATUS AND ITS IMPACT ON TREATMENT RESPONSE}

Several studies have reported that, at the time of CC diagnosis, patients are usually not undernourished ${ }^{44}$. Depending on the instrument used, undernourishment is estimated to range between $0 \%$ and $42.8 \%$ at diagnosis, and to increase up to $69 \%$ by the end of treatment with concomitant chemoradiation therapy and followed by brachytherapy. Overweight and obesity are more common, since they occur in $61.8-75.8 \%$ of patients and, simultaneously, sarcopenia is present in $10.5 \%-33.3 \% 45,46$. Furthermore, around $40.8 \%$ of women with CC have some comorbidity ${ }^{45}$, which represents a huge challenge for diagnosis and nutritional approach, since a combination of the previous diagnoses can be found in a single individual ${ }^{45}$.

\section{EFFECT OF NUTRITIONAL INTERVENTIONS ON TOXICITY AND INFLAMMATION IN CERVICAL CANCER}

An adequate diet is also indispensable as adjuvant therapy in cancer patients. Tumor cells have metabolic abnormalities that lead to high levels of reactive oxygen species, glucose metabolism changes, and micronutrient deficiencies. The ketogenic diet, which is restricted in carbohydrates and high in fat, limits glucose availability and promotes metabolic adaptations that favour oxidative phosphorilation; thus, with this type of diet enery is preferentially obtained from fatty acids. There is evidence, both clinical and in animal models, that a ketogenic diet increases oxidative stress in cancer cells, since they develop a glycolysis-dependent metabolism and are unable to obtain energy from fatty acids. Some preliminary reports of studies that are assessing the ketogenic diet as adjuvant therapy in cancer, indicate that patients who have been able to maintain the ketogenic diet for more than 3 months show improvement, tumor reduction, slowed growth, and stabilization of their physical condition. Available data indicate that cancer patients who undergo standard radiation and chemotherapy can also benefit from ketogenic diets ${ }^{47}$.

A diet low in fermentable oligosaccharides, disaccharides, monosaccharides and polyols (FODMAP) is thought to benefit patients with CC under treatment with pelvic external-beam radiotherapy. However, when investigating whether the low-FODMAP diet can decrease the severity of intestinal toxicity and improve aspects related to quality of life and decrease deterioration of performance status, no statistically significant differences were found ${ }^{48}$. Nevertheless, symptomatic and quality of life improvement has been observed in patients with chronic toxicity induced by radiotherapy ${ }^{49}$. Even so, the effects of dietary FODMAP restriction on the immune response, the production of short-chain fatty acids (SCFA), and the composition of intestinal microbiota in patients with CC, remain to be determined.

Another diet that might be beneficial for patients with $\mathrm{CC}$ that develop pelvic radiation disease (PRD), is the anti-inflammatory diet which has been tested in patients with inflammatory bowel disease (IBD), and whose symptoms are very similar to those occurring in PRD. This anti-inflammatory diet consists of five 
basic components: (1) limiting the intake of simple carbohydrates such as sugars and refined flour or processed foods; (2) eating foods with pre- and probiotics in the form of soluble fiber, such as prickly pear, banana, papaya, oatmeal, leeks, onions, and fermented foods, to restore gastrointestinal microbiota balance; (3) reducing the intake of saturated and total fats, eliminating hydrogenated oils and increasing the consumption of foods rich in omega- 3 fatty acids; (4) examining eating patterns to identify possible intolerances and detect missing nutrients; and (5) modifying the texture of food to facilitate its absorption, by cooking or grinding. Despite the difficulties following an anti-inflammatory diet, patients with IBD and good adherence have obtained improvement in symptoms ${ }^{37}$, so this could be a recommendable diet for patients with CC who are candidates for concomitant chemoradiotherapy and who are at risk of developing PRD.

Although these types of diets are promising due to their metabolic and anti-inflammatory effects, it is important to demonstrate the effect of these diets through clinical trials in CC patients, to prove their effectiveness. In conclusion, we recommend that patients with CC who are candidates for treatment based on chemoradiotherapy followed by brachytherapy, should follow a diet that meets their energyprotein requirements to avoid malnutrition, and in particular sarcopenia. Furthermore, a diet limited in simple carbohydrates and sugars, and that includes foods rich in antioxidant and anti-inflammatory nutrients, might help control intestinal inflammation and thus prevent the development of PRD and gastrointestinal toxicity.

In addition to diet, fasting has been used by many cultures throughout the history of humanity for religious and health-related reasons. A great deal of research has investigated the effects of several types of fasting and calorie restriction regimens on different tissues and the metabolism. Clinical studies have shown beneficial effects on obesity, diabetes, cardiovascular disease, autoimmune and inflammatory conditions, and cancer. In animal models, fasting reduces the development of tumors, protects the animals from treatment-related toxicity, while sensitizing the tumor to chemotherapeutics' toxicity. The mechanism involves a metabolic switch that occurs during fasting in healthy cells, which makes them resistant to stress. Cancer cells are incapable of this metabolic adaptation; thus, they become sensible to stress and unable to obtain sufficient energy. Clinical trials are underway to understand the effects of fasting and calorie restriction on several types of cancer, as reviewed elsewhere ${ }^{50}$. It will be interesting to investigate if fasting protects against chronic HPV infection and the development of CC; however, the evidence reviewed suggests that food-related factors, in particular, dietary patterns, contribute to regulate inflammation.

\section{MICROBIOTA IN CERVICAL CANCER}

The microbiota, both intestinal and vaginal, are closely linked to the immune system, and are a relevant factor associated with the development of precancerous cervical lesions in addition to HPV infection. Ninety percent of intestinal microbiota in healthy individuals is made up of Firmicutes, Bacteroidetes, Actinobacteria, Proteobacteria, and Fusobacteria. In contrast, vaginal microbiota is made up of Firmicutes, particularly Lactobacillus crispatus, Lactobacillus gasseri, Lactobacillus iners, and Lactobacillus jensenii ${ }^{51}$.

Imbalance in the microbiota composition or metabolic activity has a significant impact on an individual's health. Mechanical factors, such as vaginal douching or sexual intercourse, as well as biological factors, such as infections or the cytokine profile and the use of antibiotics, alter the vaginal microenvironment, which favors the persistence of HPV infection ${ }^{52}$. As a result, the diversity and composition of cervical microbiota differ at each stage in the natural history of CC, with an increase in microbiota diversity in CC in comparison with individuals without lesions. Lactobacillus spp. is highly abundant in the cervix of women without Pap smear abnormalities, while $L$. crispatus and $L$. iners are the most abundant species in HPVand HPV+ women without lesions, respectively. In the presence of HPV+ SIL, Sneathia spp. is the most abundant species. In late CC stages, Fusobacterium spp. is significantly more abundant than in early stages (regardless of HPV infection), and Fusobacterium necrophorum is only observed in patients with $\mathrm{CC}^{53}$. In addition, Atopobium vaginae has been associated with high-risk $\mathrm{CIN}$ and L. iners with $\mathrm{CIN} 2^{54}$.

There is a highly important association between dietary patterns and microbiota composition. In 
patients with CIN, a semi-western diet (low intake of rice, vegetables, fiber, carotenes and Vitamin C, and high intake of bread, pasta, eggs, dairy products, soft drinks, and red meat) increases the risk of CIN by favoring the establishment of $\mathrm{A}$. vaginae as the dominant species in the cervix (OR: $20.8 ; 95 \% \mathrm{Cl}: 2.21-$ $195.6 ; p=0.01)^{55}$; this suggests an association between diet and microbiota, and the susceptibility to developing CC. Finally, nutritional deficiencies have been observed to contribute to an increased risk of infection, since abnormal bacterial metabolite levels have been linked to the development of some tumors ${ }^{56,57}$.

The differences in gastrointestinal microbiota of patients with CC compared with controls have also been identified. There is an increase in Proteobacteria in patients with CC, specifically the Escherichia Shigella, Roseburia, Pseudomonas, Lachnoclostridium, Lachnospiraceae _UCG -004, Dorea, and Succinivibrio gen$\mathrm{era}^{58}$. Diet regulates the composition and function of intestinal microbiota. A high-fat diet is associated with the presence of Bacteroidetes, and a high-carbohydrate intake is associated with Prevotella. Pre- and probiotics favorably modulate the microbiota and induce preventive and therapeutic effects against cancer. The anticancer mechanisms that have been associated with the intake of pre- and probiotics include quantitative and qualitative alterations in the microbiota as well as in its metabolic capacity, inactivation of mutagenic or carcinogenic compounds, host antitumor immunity, production of anticancer, and epigenetic compounds which are involved in histone acetylation, DNA methylation, and metabolites that directly reduce intestinal inflammation ${ }^{59}$.

One of the main metabolites that have been described is butyrate, which participates in the suppression of intestinal inflammation by favoring the differentiation of regulatory $T$ cells (Treg) ${ }^{60,61}$. In murine models, a fiber-rich diet leads to a higher number of Tregs and to an increase in the production of SCFA compared to a low-fiber $\operatorname{diet}^{62}$. Butyrate restricts the synthesis of pro-inflammatory cytokines in macrophages ${ }^{63}$, suggesting a mechanism involving the innate immune response to induce tolerance to commensal microbiota and food antigens. Taken together, these data show the importance of bacterial metabolites in immune regulation, and their probable participation in the effector response against HPV infection.
In addition to modulating the immune response, there is also evidence of the contribution of microbiota to radiation therapy toxicity. Radiation-related intestinal mucosal lesions are modified by intestinal microbiota ${ }^{64-67}$. Development of toxicity is associated with changes in microbiota composition, signals derived from bacterial components, alarmins or danger-associated signals, and cytokine-mediated signals ${ }^{68}$. Patients undergoing pelvic radiotherapy show changes in microbial diversity, with a higher abundance of Proteobacteria and Gamma-proteobacteria and lower abundance of Bacteroides. The presence of Megamonas, Novosphingobium, and Prevotella, and a decrease in Bacteroides, Bacteroidaceae and Plebeius, is associated with radiotherapy. Coprococcus and Desulfovibrio are enriched before radiotherapy. There are also differential changes in microbiota abundance according to the degree of radiation enteritis (RE). In RE1 there is an abundance of Virgibacillus $(p=0.008)$, Alcanivorax ( $p=0.010)$, and Phenylobacterium $(p=0.038)$; and in RE2, abundance of Coprococcus $(p=0.044)$, Collinsella $(p=0.022)$, and rc4_4 $(p=0.020)^{69}$. Patients undergoing pelvic radiotherapy showed a higher Actinobacteria abundance and significantly less abundant Fusobacteria before treatment $^{70}$, but it changed during treatment. In gynecological and colorectal cancers, there is much evidence of microbiota involvement in tumor establishment and progression ${ }^{53,71,72}$. These observations underscore the need and possibility of finding biomarkers for early detection of the risk of developing RE and, on the other hand, finding species associated with the development of tolerance that could be used as prophylactic probiotics.

However, nutritional, dietary, and physical activity factors, which could have an impact on microbiota modulation and its effect as a risk or protective factor in the development of CC, have been poorly studied ${ }^{73}$ and, in some cases, studies have not been conclusive; therefore, further investigations are required to prove their possible interaction with the microbiota and indirectly with HPV infection and carcinogenesis.

Probiotics are a mechanism to attempt to restore homeostasis in the microbiota. A pilot study examined the effect of probiotics in women with HPV and $\mathrm{LSIL}^{74}$. Fifty-four women were randomized to consume probiotics (commercial fermented dairy beverage with Lactobacillus casei Shirota) or to follow 
standard care for 6 months (without treatment and with the indication not to consume probiotics); $50 \%$ of the women in the intervention group had a negative result for intraepithelial lesion compared to $29.6 \%$ of women in the control group.

A series of randomized, double-blind, and placebocontrolled trials have assessed in detail the effect of probiotics. In locally advanced CC, a probiotic containing Lactobacillus acidophilus plus Bifidobacterium bifidum (Infloran) decreased the incidence of diarrhea when patients underwent radiotherapy concomitantly with cisplatin (45\% in the placebo group and $9 \%$ in the intervention group). During follow-up, the use of antidiarrheal drugs significantly decreased in the intervention group ( $68 \%$ in placebo vs. $91 \%$ in study group, $p=0.03$ ), while patients in the study group had a significant improvement in stool consistency (35\% in placebo vs. $81 \%$ in study group, $p=0.001)^{75}$. In patients undergoing radiation of pelvic tumors with or without chemotherapy, supplementation with Bifilact double strain probiotics (L. acidophilus LAC-361 and Bulgaricus longum BB-536), resulted in a decrease in diarrhea severity in $35 \%$ of patients in the group that received the probiotic, in comparison with only $17 \%$ of patients in the placebo group $(p=0.04)^{64}$. In sigmoid, rectal or CC, patients who received the VSL \# 3 probiotic (L. casei, Lactobacillus plantarum, L. acidophilus, Lactobacillus delbrueckii spp. Bulgaricus, $B$. longum, Bulgaricus breve, Bulgaricus infantis, and Streptococcus salivarius sub sp. thermophilus), on the $1^{\text {st }}$ day of radiotherapy, had significantly less diarrhea than those who received placebo $(31.6 \%$ in the probiotic group vs. $51.8 \%$ in the placebo, $p=0.001$ ), and the severity of diarrhea in patients receiving the probiotic was significantly lower $(55.4 \%$ in the placebo group vs. 1.4 in the probiotic group, $p=0.001$ ). In addition, the number of daily bowel movements in the placebo group was $14.7 \pm 6$ in comparison with $5.1 \pm$ 3 in the probiotic group $(p=0.05)^{76}$. In cervical carcinoma or endometrial adenocarcinoma, a probiotic beverage with $L$. casei $\mathrm{DN}-114$ 001, did not significantly decrease the incidence of radiation-induced diarrhea or the use of antidiarrheal medication ( $p=0.568$ ), but patients did report significantly better stool consistency $(p=0.04)^{77}$. In gynecological cancer patients, a prebiotic ( $50 \%$ inulin $+50 \%$ fructo-oligosaccharides) protected from gastrointestinal radiotherapy-induced toxicity ${ }^{65}$. Taken together, the results of these studies indicate that probiotics can mitigate radiation-induced toxicity in patients with gynecological cancer who receive radiation therapy to the pelvic area.

Several recent studies have shown that microbiota plays a role in the response to immunotherapy. Microbiota and its metabolites alter IL-12 and toll-like receptor (TLR2)/TLR4 signaling pathways, which results in CTLA-4 ${ }^{78,79}$ and PD-L1 blockade ${ }^{80,81}$. Therefore, the intestinal microbiome is an important clinical marker and a therapeutic target for immunotherapy in CC.

\section{CONCLUSIONS}

The relationship between diet, nutritional status, and microbiota shows that prevention and treatment in CC should be comprehensive, to provide better strategies for the prevention, diagnosis, and prediction of the risk of recurrence. Treatment with diet, pre- and probiotics should be routinely administered to modulate the immune system that contributes to a decreased risk of HPV infection and CC development and, in patients with CC, prevents treatment-associated inflammation and toxicity, thus improving the patients' quality of life.

\section{RECOMMENDATIONS}

1. The consumption of a prudent dietary pattern, rich in vegetables, fruits, whole grains, omega- 3 fatty acids, and limited in red meats, saturated fatty acids, and scarce in sugar and refined processed products is recommended, since it is associated with an optimal immune function, tolerogenic to the commensal microbiota and food antigens, and responsive to potential infectious pathogens. This dietary pattern is also associated with a high antioxidant status capable of neutralizing the redox state and preventing carcinogenesis. Quality of evidence: (GRADE) Moderate. Strength of recommendation: Strong in favor of its use.

2. The consumption of probiotic supplements during treatment with pelvic radiotherapy is recommended because it is associated to a decrease in gastrointestinal symptom severity and frequency. Quality of evidence: (GRADE) Low. Strength of recommendation: Weak in favor of its use. 


\section{REFERENCES}

1. Albers R, Bourdet-Sicard R, Braun D, Calder PC, Herz U, Lambert $C$, et al. Monitoring immune modulation by nutrition in the general population: identifying and substantiating effects on human health. Br J Nutr. 2013:110:S1-30.

2. Soldati L, Di Renzo L, Jirillo E, Ascierto PA, Marincola FM, De Lorenzo A. The influence of diet on anti-cancer immune responsiveness. J Transl Med. 2018;16:75.

3. Fraker P. Impact of nutritional status on immune integrity. In: Nutrition and Immunology. Totowa, NJ: Humana Press; 2000. p.147-56

4. Chandra RK. Nutrition and the immune system: an introduction. Am J Clin Nutr. 1997;66:460S-3.

5. Geuking MB, Köller Y, Rupp S, McCoy KD. The interplay between the gut microbiota and the immune system. Gut Microbes. 2014:5:411-8.

6. Yip C, Dinkel C, Mahajan A, Siddique M, Cook GJ, Goh V. Imaging body composition in cancer patients: visceral obesity, sarcopenia and sarcopenic obesity may impact on clinical outcome. Insights Imaging. 2015;6:489-97.

7. Poorolajal J, Jenabi E. The association between BMI and cervical cancer risk: a meta-analysis. Eur J Cancer Prev. 2016;25:232-8.

8. Gu W. Obesity-associated endometrial and cervical cancers. Front Biosci. 2013;E5:E600.

9. Tsoli M, Robertson G. Cancer cachexia: malignant inflammation, tumorkines, and metabolic mayhem. Trends Endocrinol Metab. 2013;24:174-83

10. García-Closas R, Castellsagué X, Bosch X, González CA. The role of diet and nutrition in cervical carcinogenesis: a review of recent evidence. Int J Cancer. 2005:117:629-37.

11. Giuliano AR, Siegel EM, Roe DJ, Ferreira S, Baggio ML, Galan L, et al. Dietary intake and risk of persistent human papillomavirus (HPV) infection: the ludwig-McGill HPV natural history study. J Infect Dis. 2003;188:1508-16.

12. Lopes RD, Teixeira JA, Marchioni D, Villa LL, Giuliano AR, Baggio $M L$, et al. Dietary intake of selected nutrients and persistence of HPV infection in men. Int J Cancer. 2017:141:757-65.

13. Couto E, Boffetta P, Lagiou P, Ferrari P, Buckland G, Overvad K, et al. Mediterranean dietary pattern and cancer risk in the EPIC cohort. Br J Cancer. 2011:104:1493-9.

14. Barchitta M, Maugeri A, Quattrocchi A, Agrifoglio O, Scalisi A, Agodi A. The association of dietary patterns with high-risk human papillomavirus infection and cervical cancer: a cross-sectional study in Italy. Nutrients. 2018:10:E469.

15. Giuliano AR, Papenfuss M, Nour M, Canfield LM, Schneider A, Hatch K. Antioxidant nutrients: associations with persistent human papillomavirus infection. Cancer Epidemiol Biomarkers Prev. 1997;6:917-23.

16. Piyathilake CJ, Macaluso M, Brill I, Heimburger DC, Partridge EE. Lower red blood cell folate enhances the HPV-16-associated risk of cervical intraepithelial neoplasia. Nutrition. 2007; 23:203-10.

17. Chih HJ, Lee AH, Colville L, Binns CW, Xu D. A review of dietary prevention of human papillomavirus-related infection of the cervix and cervical intraepithelial neoplasia. Nutr Cancer. 2013; 65:317-28.

18. Leischner C, Burkard M, Pfeiffer MM, Lauer UM, Busch C, Venturelli S. Nutritional immunology: function of natural killer cells and their modulation by resveratrol for cancer prevention and treatment. Nutr J. 2015;15:47.

19. Tomita LY, Filho AL, Costa MC, Andreoli MA, Villa LL, Franco EL, et al. Diet and serum micronutrients in relation to cervical neoplasia and cancer among low-income Brazilian women. Int J Cancer. 2010;126:703-14

20. Tomita LY, Roteli-Martins CM, Villa LL, Franco EL, Cardoso MA BRINCA Study Team. Associations of dietary dark-green and deep-yellow vegetables and fruits with cervical intraepithelial neoplasia: modification by smoking. Br J Nutr. 2011;105: 928-37.

21. Xiao S, Tang YS, Kusumanchi P, Stabler SP, Zhang Y, Antony AC. Folate deficiency facilitates genomic integration of human papillomavirus Type 16 DNA in vivo in a novel mouse model for rapid oncogenic transformation of human keratinocytes. J Nutr. 2018;148:389-400.

22. Asemi Z, Vahedpoor Z, Jamilian M, Bahmani F, Esmaillzadeh A. Effects of long-term folate supplementation on metabolic status and regression of cervical intraepithelial neoplasia: a randomized, double-blind, placebo-controlled trial. Nutrition. 2016; 32:681-6.
23. González CA, Travier N, Luján-Barroso L, Castellsagué X, Bosch FX, Roura E, et al. Dietary factors and in situ and invasive cervical cancer risk in the European prospective investigation into cancer and nutrition study. Int J Cancer. 2011;129:449-59.

24. Kjellberg L, Hallmans G, Ahren AM, Johansson R, Bergman F, Wadell G, et al. Smoking, diet, pregnancy and oral contraceptive use as risk factors for cervical intra-epithelial neoplasia in relation to human papillomavirus infection. $\mathrm{Br}$ J Cancer. 2000; $82: 1332-8$

25. Divya CS, Pillai MR. Antitumor action of curcumin in human papillomavirus associated cells involves downregulation of viral oncogenes, prevention of NFkB and AP-1 translocation, and modulation of apoptosis. Mol Carcinog. 2006;45:320-32.

26. Maher DM, Bell MC, O’Donnell EA, Gupta BK, Jaggi M, Chauhan SC. Curcumin suppresses human papillomavirus oncoproteins, restores $\mathrm{p} 53$, rb, and ptpn 13 proteins and inhibits benzo[a]pyreneinduced upregulation of HPV E7. Mol Carcinog. 2011;50:47-57.

27. Chen AL, Hsu CH, Lin JK, Hsu MM, Ho YF, She TS, et al. Phase clinical trial of curcumin, a chemopreventive agent, in patients with high-risk or pre-malignant lesions. Anticancer Res. 2001; 21:2895-900.

28. Prusty BK, Das BC. Constitutive activation of transcription factor AP-1 in cervical cancer and suppression of human papillomavirus (HPV) transcription and AP-1 activity in HeLa cells by curcumin. Int ] Cancer 2005:113:951-60.

29. Di Domenico F, Foppoli C, Coccia R, Perluigi M. Antioxidants in cervical cancer: chemopreventive and chemotherapeutic effects of polyphenols. Biochim Biophys Acta Mol Basis Dis. 2012; 1822:737-47.

30. Ahn WS, Yoo J, Huh SW, Kim CK, Lee JM, Namkoong SE, et al. Protective effects of green tea extracts (polyphenon $\mathrm{E}$ and EGCG) on human cervical lesions. Eur J Cancer Prev. 2003. $12: 383-90$

31. Siegel EM, Salemi JL, Villa LL, Ferenczy A, Franco EL, Giuliano AR. Dietary consumption of antioxidant nutrients and risk of incident cervical intraepithelial neoplasia. Gynecol Oncol. 2010; 118:289-94

32. Feng CY, Lin M, Lakhaney D, Sun HK, Dai XB, Zhao FH, et al. The association between dietary intake and cervical intraepithelial neoplasia grade 2 or higher among women in a high-risk rural area of China. Arch Gynecol Obstet. 2011;284:973-80.

33. Hwang $\mathrm{JH}$, Lee JK, Kim TJ, Kim MK The association between fruit and vegetable consumption and HPV viral load in high-risk HPV-positive women with cervical intraepithelial neoplasia. Cancer Causes Control. 2010;21:51-9.

34. Minihane AM, Vinoy S, Russell WR, Baka A, Roche HM, Tuohy $\mathrm{KM}$, et al. Low-grade inflammation, diet composition and health: current research evidence and its translation. $\mathrm{Br}$ J Nutr. 2015; 114:999-1012.

35. Ostan R, Lanzarini C, Pini E, Scurti M, Vianello D, Bertarelli C, et al. Inflammaging and cancer: a challenge for the Mediterranean diet. Nutrients. 2015;7:2589-621.

36. De Almeida CV, de Camargo MR, Russo E, Amedei A. Role of diet and gut microbiota on colorectal cancer immunomodulation. World J Gastroenterol. 2018;25:151-62.

37. Mantzioris E, Cleland LG, Gibson RA, Neumann MA, Demasi M, James MJ. Biochemical Effects of a Diet Containing Foods Enriched with N-3 Fatty Acids. Am J Clin Nutr. 2000;72:42-8

38. Varela-Moreiras G, Avila JM, Cuadrado C, del Pozo S, Ruiz E, Moreiras $\mathrm{O}$. Evaluation of food consumption and dietary patterns in Spain by the food consumption survey: updated information. Eur J Clin Nutr. 2010;64:S37-43.

39. Argyriou AA, Chroni E, Koutras A, Iconomou G, Papapetropoulos $\mathrm{S}$, Polychronopoulos $\mathrm{P}$, et al. A randomized controlled trial evaluating the efficacy and safety of Vitamin E supplementation for protection against cisplatin-induced peripheral neuropathy: final results. Support Care Cancer. 2006:14:1134-40.

40. Gourineni VP, Verghese M, Boateng J, Shackelford L, Bhat NK, Walker LT. Combinational effects of prebiotics and soybean against azoxymethane-induced colon cancer in vivo. J Nutr Metab. 2011;2011:868197.

41. Hijova E, Szabadosova V, Strojny L, Bomba A. Changes chemopreventive markers in colorectal cancer development after inulin supplementation. Bratislava Med J. 2014:115:76-9.

42. Ghosh C, Baker JA, Moysich KB, Rivera R, Brasure JR, McCann SE. Dietary intakes of selected nutrients and food groups and risk of cervical cancer. Nutr Cancer. 2008;60:331-41.

43. Hosono S, Matsuo K, Kajiyama H, Hirose K, Suzuki T, Kawase T, et al. Association between dietary calcium and Vitamin D intake and cervical carcinogenesis among Japanese women. Eur J Clin Nutr. 2010;64:400-9. 
44. Laky B, Janda M, Bauer J, Vavra C, Cleghorn G, Obermair A. Malnutrition among gynaecological cancer patients. Eur J Clin Nutr. 2007;61:642-6.

45. Aredes MA, Garcez MR, Chaves GV. Influence of chemoradiotherapy on nutritional status, functional capacity, quality of life and toxicity of treatment for patients with cervical cancer. Nutr Diet. 2018;75:263-70.

46. Sánchez M, Castro-Eguiluz D, Luvián-Morales J, Jiménez-Lima R, Aguilar-Ponce JL, Isla-Ortiz D, et al. Deterioration of nutritional status of patients with locally advanced cervical cancer during treatment with concomitant chemoradiotherapy. J Hum Nutr Diet. 2019;32:480-91.

47. Allen BG, Bhatia SK, Anderson CM, Eichenberger-Gilmore JM, Sibenaller ZA, Mapuskar KA, et al. Ketogenic diets as an adjuvant cancer therapy: history and potential mechanism. Redox Biol. 2014;2:963-70.

48. Soto-Lugo JH, Souto-Del-Bosque MA, Vázquez Martínez CA. Effectiveness of a nutritional intervention in the reduction of gastrointestinal toxicity during teletherapy in women with gynaecological tumours. Rev Méd Hosp Gen México. 2018;81:7-14.

49. Larsen T, Hausken T, Ystad SO, Hovdenak N, Mueller B, Lied GA Does the low FODMAP diet improve symptoms of radiation-induced enteropathy? A pilot study. Scand J Gastroenterol. 2018; 53:541-8.

50. De Cabo R, Mattson MP. Effects of intermittent fasting on health, aging, and disease. N Engl J Med. 2019;381:2541-51.

51. Schwabe RF, Jobin C. The microbiome and cancer. Nat Rev Cancer. 2013;13:800-12.

52. Gao W, Weng J, Gao Y, Chen X. Comparison of the vaginal microbiota diversity of women with and without human papillomavirus infection: a cross-sectional study. BMC Infect Dis. 2013; 13:271.

53. Audirac-Chalifour A, Torres-Poveda K, Bahena-Román M, TéllezSosa J, Martínez-Barnetche J, Cortina-Ceballos B, et al. Cervical microbiome and cytokine profile at various stages of cervical cancer: a pilot study. PLoS One. 2016;11:e0153274

54. Piyathilake CJ, Ollberding NJ, Kumar R, Macaluso M, Alvarez RD, Morrow CD. Cervical microbiota associated with higher grade cervical intraepithelial neoplasia in women infected with highrisk human papillomaviruses. Cancer Prev Res. 2016;9:357-66.

55. Seo SS, Oh HY, Lee JK, Kong JS, Lee DO, Kim MK. Combined effect of diet and cervical microbiome on the risk of cervical intraepithelial neoplasia. Clin Nutr. 2016;35:1434-41.

56. Serban DE. Gastrointestinal cancers: influence of gut microbiota, probiotics and prebiotics. Cancer Lett. 2014:345:258-70.

57. Kau AL, Ahern PP, Griffin NW, Goodman AL, Gordon Jl. Human nutrition, the gut microbiome and the immune system. Nature. 2011;474:327-36

58. Wang Z, Wang $Q$, Zhao J, Gong L, Zhang Y, Wang X, et al. Altered diversity and composition of the gut microbiome in patients with cervical cancer. AMB Express. 2019;9:40.

59. Plottel CS, Blaser MJ. Microbiome and malignancy. Cell Host Microbe. 2011;10:324-35

60. Furusawa Y, Obata Y, Fukuda S, Endo TA, Nakato G, Takahashi D, et al. Commensal microbe-derived butyrate induces the differentiation of colonic regulatory T cells. Nature. 2013;504:446-50.

61. Singh N, Gurav A, Sivaprakasam S, Brady E, Padia R, Shi H, et al. Activation of Gpr109a, receptor for niacin and the commensal metabolite butyrate, suppresses colonic inflammation and carcinogenesis. Immunity. 2014:40:128-39.

62. Arpaia N, Campbell C, Fan X, Dikiy S, Van Der Veeken J, Deroos $\mathrm{P}$, et al. Metabolites produced by commensal bacteria promote peripheral regulatory $\mathrm{T}$-cell generation. Nature. 2013;504:451-5.

63. Chang PV, Hao L, Offermanns S, Medzhitov R. The microbial metabolite butyrate regulates intestinal macrophage function via histone deacetylase inhibition. Proc Natl Acad Sci U S A. 2014;111:2247-52.
64. Demers M, Dagnault A, Desjardins J. A randomized double-blind controlled trial: impact of probiotics on diarrhea in patients treated with pelvic radiation. Clin Nutr. 2014;33:761-7.

65. García-Peris P, Velasco C, Lozano MA Moreno Y, Paron L, de la Cuerda $C$, et al. Effect of a mixture of inulin and fructo-oligosaccharide on Lactobacillus and Bifidobacterium intestinal microbiota of patients receiving radiotherapy: a randomised, doubleblind, placebo-controlled trial. Nutr Hosp. 2012;27:1908-15.

66. McQuade JL, Daniel CR, Helmink BA, Wargo JA. Modulating the microbiome to improve therapeutic response in cancer. Lancet Oncol. 2019:20:e77-91.

67. Linn $\mathrm{YH}$, Thu KK, Win NH. Effect of probiotics for the prevention of acute radiation-induced diarrhoea among cervical cancer patients: a randomized double-blind placebo-controlled study. Probiotics Antimicrob Proteins. 2019;11:638-47.

68. Sokol H, Adolph TE. The microbiota: an underestimated actor in radiation-induced lesions? Gut. 2018;67:1-2.

69. Wang Z, Wang Q, Wang X, Zhu L, Chen J, Zhang B, et al. Gut microbial dysbiosis is associated with development and progression of radiation enteritis during pelvic radiotherapy. ] Cell Mol Med. 2019;23:3747-56.

70. Nam Y Do, Kim HJ, Seo JG, Kang SW, Bae JW. Impact of pelvic radiotherapy on gut microbiota of gynecological cancer patients revealed by massive pyrosequencing. PLoS One. 2013;8:1-11.

71. Rubinstein MR, Wang X, Liu W, Hao Y, Cai G, Han YW. Fusobacterium nucleatum promotes colorectal carcinogenesis by modulating E-cadherin/ $\beta$-catenin signaling via its FadA adhesin. Cell Host Microbe. 2013;14:195-206.

72. Chase D, Goulder A, Zenhausern F, Monk B, Herbst-Kralovetz M. The vaginal and gastrointestinal microbiomes in gynecologic cancers: a review of applications in etiology, symptoms and treatment. Gynecol Oncol. 2015;138:190-200.

73. Latino-Martel P, Cottet V, Druesne-Pecollo N, Pierre FH, Touillaud $M$, Touvier $M$, et al. Alcoholic beverages, obesity, physical activity and other nutritional factors, and cancer risk: a review of the evidence. Crit Rev Oncol Hematol. 2016;99:308-23.

74. Verhoeven V, Renard N, Makar A, Van Royen P, Bogers JP, Lardon $\mathrm{F}$, et al. Probiotics enhance the clearance of human papillomavirus-related cervical lesions: a prospective controlled pilot study. Eur J Cancer Prev. 2013;22:46-51.

75. Chitapanarux I, Chitapanarux T, Traisathit P, Kudumpee S, Tharavichitkul E, Lorvidhaya $\mathrm{V}$. Randomized controlled trial of live Lactobacillus acidophilus plus Bifidobacterium bifidum in prophylaxis of diarrhea during radiotherapy in cervical cancer patients. Radiat Oncol. 2010;5:31.

76. Delia P, Sansotta G, Donato V, Frosina P, Messina G, De Renzis $C$, et al. Use of probiotics for prevention of radiation-induced diarrhea. World J Gastroenterol. 2007;13:912-5.

77. Giralt ], Regadera JP Verges R, Romero ], de la Fuente I, Biete A, et al. Effects of probiotic Lactobacillus casei DN-114 001 in prevention of radiation-induced diarrhea: results from multicenter, randomized, placebo-controlled nutritional trial. Int J Radiat Oncol Biol Phys. 2008:71:1213-9.

78. Vétizou M, Pitt JM, Daillère R, Lepage $P$, Waldschmitt $N$, Flament $C$, et al. Anticancer immunotherapy by CTLA-4 blockade relies on the gut microbiota. Science. 2015;350:1079-84

79. Pitt JM, Vétizou M, Boneca IG, Lepage P, Chamaillard M, Zitvogel L. Enhancing the clinical coverage and anticancer efficacy of immune checkpoint blockade through manipulation of the gut microbiota. Oncoimmunology. 2017;6:e1132137.

80. Sivan A, Corrales L, Hubert N, Williams JB, Aquino-Michaels K, Earley ZM, et al. Commensal Bifidobacterium promotes antitumor immunity and facilitates anti-PD-L1 efficacy. Science. 2015;350:1084-9.

81. Routy B, Le Chatelier E, Derosa L, Duong CP, Alou MT, Daillère $R$, et al. Gut microbiome influences efficacy of PD-1-based immunotherapy against epithelial tumors. Science. 2018; 359:91-7. 